\title{
Analysis and Performance Evaluation of Selective Channel Assignment Method in Cognitive Radio System
}

\author{
Mallikarjuna Gowda C. $\mathrm{P}^{1}$ and Vijayakumar $\mathrm{T}^{2}$ \\ ${ }^{1}$ Department of Electronics and Communications Engineering, SJB Institute of \\ Technology, faculty at BMS Institute of Technology and Management, Bengaluru, \\ Affiliated to Visvesvaraya Technological University, Belagavi, India. \\ ${ }^{2}$ Department of Electronics and Communications Engineering, SJB Institute of Technology, \\ Bengaluru, Affiliated to Visvesvaraya Technological University, Belagavi, India.
}

\section{ABSTRACT}

An issue of realizing dynamic spectrum access in cognitive radio network is the design of an efficient channel assignment mechanism. In this work, a selective channel assignment method is proposed to divide the channels between the Primary Users (Pus) and Secondary Users (SUs). There is need to build two first fit assignment models in one single model to treat each of the PU and SUs separately. Also, there should be a flexibility to decide which part of the channel to be centered around for the allocation of PU and SUs, an attempt is made to design such a model. The performance of the model is tested on the network that has converters and also on network that does not have any converters installed. The percentage of PU calls over all the calls is also varied to determine the change in blocking probabilities. Extensive simulation results show that the proposed selective channel assignment method performs better with respect to performance parameters such as the blocking probabilities, throughput and the channel usage frequency, when compared with the existing channel assignment methods such as first fit assignment and uniformly distributed random assignment methods.

KEY WORDS: THROUGHPUT, COGNITIVE RADIO NETWORKS, BLOCKING PROBABILITY, QUALITY-OF-SERVICE (QOS), CHANNEL ASSIGNMENT.

\section{INTRODUCTION}

People's approach of life and thinking are changing due to exponential growth of wireless devices and applications. Also, the demand for electromagnetic radio spectrum has increased. Cognitive radio technology has been proposed as an operational way to facilitate dynamic spectrum access (DSA) to increase spectrum utilization efficiency

\section{ARTICLE INFORMATION}

*Corresponding Author: cpmallikarjunagowda@bmsit.in Received 11th Oct 2020 Accepted after revision 29th Dec 2020 Print ISSN: 0974-6455 Online ISSN: 2321-4007 CODEN: BBRCBA

Thomson Reuters ISI Web of Science Clarivate Analytics USA and Crossref Indexed Journal

\section{Clarivate
Analytics}

NAAS Journal Score 2020 (4.31)

A Society of Science and Nature Publication,

Bhopal India 2020. All rights reserved.

Online Contents Available at: http//www.bbrc.in/

Doi: http://dx.doi.org/10.21786/bbrc/13.13/26 in cognitive radio networks (CRN). The kinds of nodes in cognitive radio network are known as licensed primary users (PUs) and unlicensed cognitive or secondary users (SUs). Cognitive radio (CR) enables the radio devices with the perception capability to learn the surrounding radio environment and adjust their configurations to increase the spectrum utilization.

According to the predictions study made by Cisco on global wireless networking metrics, by 2023, there will be 5.7 billion more mobile users, 13.1 billion more mobile connections and faster mobile speeds of $43.9 \mathrm{Mbps}$, when compared to 5.1 billion users, 8.8 billion mobile connections and 13.2 Mbps mobile speeds in the year 2018. Rajeev Ranjan, et.al.(2020), have explored thoughtprovoking features of co-channel and adjacent channel interference to increase the performance of the network and to provide QoS to both primary and secondary nodes 
in a cognitive radio network. Using interference index as an important feature, reduced co-channel interference among secondary nodes, which indirectly impacts adjacent channel interference to them.

By limiting the interference lower than the acceptance threshold of $10 \mathrm{dBm}$ to primary users, the concept of interference index with the distributed greedy algorithm is successful in maximising the cognitive radio network capacity with an average value 60\%. Secondary users transmit power always maintained lower than the primary transmit power of $30 \mathrm{dBm}$ as a pride of primary users. Also, interference index can be used to deal statistically by experimenting at algorithm level as interference management, with high traffic loads, during emergency services and disaster reliefs, to discuss between the mobility of the secondary users in cognitive radio network and channel vacating parameter as interference limit indicator as a future work.

Yousuf Aborahama and Mohamed S. Hassan (2020), have inspected in cognitive radio networks, the extent of time with which a secondary user can hold a primary channel and the average quantity of data that can be transmitted. To emulate reality, the activity levels of primary users and secondary users, the primary channels identical and non-identical distribution of time were derived in closed form probabilistic expressions. Haythem Bany Salameh, et.al.(2020), developed an in-band full-duplex(IB-FD) routing protocol, which aims at maximizing the endto-end network throughput subject to interference constraints for a given set of routes among a cognitive radio source destination pair.

Xiukui Li and Seyed A. (Reza) Zekavat (2009), estimated the PU and SU traffic by using a prediction algorithm. The probability of allocation of a channel to a SU was predicted and it was related to the channels meant for PUs. However, these methods have higher computational complexity. There is also another methodology by X. Li S.A. (Reza) Zekavat (2010), proposed to predict the PU traffic. The research was focused on the methods to improve the predictability of PU traffic. Cooperative spectrum sharing was also proposed by some researchers. Cooperative spectrum sharing involves sharing of static CR nodes of various service providers proposed by $R$. Kaniezhil and C. Chandrasekar (2012).

Muhammad Rehan Usman, et.al (2017), have proposed, two channel assignment techniques, in variable channel assignment method (VCAM), based on either time sharing or interference created by secondary nodes, variable sharing can be adjusted dynamically and in reserved variable channel assignment method (RVCAM), channels are reserved separately for primary users. The blocking probability of the primary users and secondary users are managed dynamically by using channel sharing. According to the traffic conditions, by regulating the channel sharing between primary and secondary nodes, blocking probability of the primary users can be controlled. The drawback of the reserved variable channel assignment method is secondary users cannot use the channels which are reserved for primary users, when they are not in use also.

Vamsi Krishna Tumuluru, et. al (2013), have investigated the cognitive radio network with centralized and distributed architectures for the prioritized unlicensed users traffic by reserving sub-channels and the spectrum hand-off prioritization using dynamic spectrum access systems. For performance evaluation, the parameters considered are the call completion rate and the mean handoff delay for the two priority classes in the secondary node traffic, blocking probability and forced termination probability. Sandeep Mavuduru Kannappa and Mohammad Saquib (2010), proposed a novel spectrum assignment scheme to reduce the call dropping probability of the secondary users in cognitive wireless networks by dynamically assigning the service rates to the secondary nodes depending on the existing network spectral resource. Developed an 2D Markov chain to analyse the spectrum sharing policies, by increasing their maximum allowable service rates, lower call dropping probability of six to seven times is achieved by the proposed scheme when compared to the existing one.

Yan Zhang (2008), proposed and analyzed a new dynamic spectrum access scheme to avoid direct blocking of secondary node with and without buffering in cognitive radio wireless networks. Developed a Markov approach to analyse for both primary and secondary system, spectrum sharing policies based on bandwidth size. Also, forced termination probability, blocking probability, non-completion probability, interrupted probability and waiting time as performance metrics for secondary node and it is found that the buffer is able to decrease the secondary node blocking probability and non-completion probability with small rise in forced termination probability. Amir Sepasi Zahmati, et.al (2009), developed a model for $\mathrm{N}$ secondary users and one primary user to analyse spectrum usage in a heterogeneous cognitive radio network.

Authors Zeljko Tabakovic and Mislav Grgic (2016), have addressed the frequency assignment issue in the cognitive radio networks. Authors have treated the frequency assignment issue in cognitive radio network as a graph coloring problem. In the frequency assignment decision process instead of channel selection authors have proposed frequency and bandwidth selection. An objective minimizing network interference and maximizing network throughput is achieved by assigning channels to secondary nodes in cognitive radio network through the use of centralized and distributed sequential algorithms. It is possible to compute the individual interference mechanisms and cumulative interference with the use of interference weighting and categorization scheme which is useful to the cognitive radio network performance and results in more efficient spectrum usage and a reduced mutual influence between terminals of around 2.5 -12 times less when compared with binary interference model. 
Murtaza Zafer and Eytan Modiano (2006), established a novel channel assignment algorithm that decreases blocking probability by spatially re-using the frequencies in a well-organized way. Also deliberated on the blocking probability behaviour of the connection oriented traffic for multi-hop wireless line and grid topologies by focussing on the influence of communication range of the nodes. It is shown that the line topology using a large communication range significantly decreases blocking probability with the dynamic channel assignment algorithm.

In methodologies proposed by I. Ketzela, M. Nagheshineh (1996), S.K. Das, S.K. Sen, R. Jayaram (1996), H.Jiang, S. Rappaport (1996), J.C.I. Chuang, R. Mathar (1993), J. Mattfeldt, G. Cao, M. Singhal (1993), S. A. El-Dolil, W. C. Wong, and R. Steele (1989), channel assignment was performed at a central location. The centralized channel allocation can be made with a mobile switching center. The mobile switching center has all the details about how and when a particular channel was used and its current status. The current status is obtained by the mobile switching center by getting the information from the local networks as soon as a channel was assigned to a PU or SU call. With this method, the mobile switching center has all the required information to avoid interference of the calls there by reducing the blocking probabilities to minimum or zero. In case the mobile switching center fails, then it leaves a chaotic situation and the network will go out of control in terms of interference. Hence proper care and maintenance should be undertaken on periodic basis to avoid the single fault failure of the centralized channel allocation system.

To overcome the above problem, many designs of decentralized channel allocation system have been proposed. The decentralized channel allocation system does not suffer the drawback of single fault failure. The entire cognitive radio system is divided into cells and each cell is equipped with a base station. The purpose of a base station in a cell is to manage the PU and SU traffic of that cell. The base station allocates a channel based on the information present at the point of time about that particular node. It will not have any dependency from other cells while allocating the channels and completely dependent only on the local information about the channel's status. There is also a model where the channels of one cell are allocated by base stations of other cells. In such a case, whenever as channel of a cell is assigned by a base station; it informs the decision to allocate a channel to all other base stations so that other base stations will not assign the same channel.

In this work, three channel allocation methods are discussed. All the allocations are carried out in a base station of a cell. The channel assignment methods like first fit, random assignment and selective assignment methods are implemented and compared. The selective channel assignment specifically suitable for a case like cognitive radio system where there is more than one type of calls in the network. As per the literature analysis, it is observed that most of the channel assignment methods are either first fit or uniformly distributed channel assignment methods. The first fit assignment is much superior to random assignment method. But the first fit assignment also leads to $100 \%$ blocking probability in some cases. Therefore, there is a need to develop a new channel assignment method that reduces the blocking probability. This is very essential for the case of cognitive radio system where there are two types of calls, namely, PU and SUs.

In this work, a new channel assignment, namely, selective channel assignment method is proposed to divide the channels between the PU and SUs. In other words, there is need to build two first fit assignment models in one single model to treat each of the PU and SUs separately. Also, there should be a flexibility to decide which part of the channel to be centered around for the allocation of PU and SUs. In this work, an attempt is made to design such a model. The performance of the model is tested on the network that has converters and also on network that does not have any converters installed. The percentage of PU calls over all the calls is also varied to determine the change in blocking probabilities. In the next section, details of selective channel assignment method are discussed. In Section III, simulation results are presented for cases like with and without conversions, type of channel assignment method and percentage of PU calls. Finally, in Section IV, the conclusions are presented.

II. Selective Channel Assignment: In selective channel assignment, when a call arrives, it will be assessed if it is a PU call or an SU call. In case if it is a PU call then that call is assigned to the lower order nodes and if it is an SU call, it is assigned to higher order nodes. There will be some channels around the chosen center nodes both for PU and SU calls. For example, if the channel 2 is chosen as center node for PU, then nodes 1, 3 and 4 are the surrounding nearby nodes. All the PU calls will be assigned to channel 2 first if it is free and to channel 1 or 3 if 2 is not free. Then if channels 1,2 and 3 are busy, then channel 4 is assigned. If channels 2, 3 and 4 are busy and if channel 1 becomes free, then channel 1 is assigned to the next new call. This way only freely available channels in the lower order are utilized for allocation. Similarly, when an SU arrives, if the center node chosen for SU call is 12, then nodes 13,14 and 15 are the surrounding nodes. In this work, channels 1 and 15 are chosen as the center nodes. The algorithm has the flexibility to choose the center node. Higher the gap between the center nodes between PU and SU, lower the blocking probability and higher the throughput.

2.1 Selective Channel assignment algorithm with and without conversion: The algorithm for the selective channel assignment is provided below for conversion and no conversion of wavelength.

Step 1: Initialize the variables OCC-CHAN-TIME $=0$, CHAN-USG-FREQ $=0$ and CNT-CAL-BLK $=0$.

Step 2: Initialize the selective channel algorithm variables CENTER-POINT $=0.1$ and SPREAD $=0.1$. 
Step 3: Determine if arrived call is a PU or SU.

Step 4: Identify free channels in the first link.

Step 5: Arrange free channels in ascending order.

Step 6: Generate random number between 0 and 1 using Gaussian distribution with mean $=$ CENTER-POINT, standard deviation $=$ SPREAD.

Step 7: If call is a PU, then Channel = Number of channels * random number

Step 8: If call is an SU, then Channel = Number of channels - [Number of channels * random number]

Step 9: Assign the selected channel to the call

Step 10: Update CHAN-USG-FREQ.= CHAN-USG-FREQ $+1$

Step 11: Update OCC-CHAN-TIME = TIME-HOLD-CALL

Step 12: DIFF = OCC-CHAN-TIME - TIME-NEXT-CALL

Step 13: If DIFF > 0, then OCC-CHAN-TIME $=$ OCCCHAN-TIME - TIME-NEXT-CALL for the next call.

Step 14: If DIFF $<=0$ then OCC-CHAN-TIME $=0$.

Step 15: If the first link free, then assign the next call, else call is blocked.

Step 16: Check all the previous calls and if the next link is not free, the call is blocked.

Step 17: If the first link free, then assign the next call, else find the free channel in the first link.

Step 18: Convert the wavelength to the wavelength of free channel and assign the call.

Step 19: If all the channels are busy in the first link, then call is blocked.

Step 20: Check all the previous calls and if the next link is not free, find the free channel in the next link

Step 21: Convert the wavelength to the wavelength of free channel in the next link.

Step 22: If all the channels are busy in the next link, then call is blocked.

Step 23: Update CNT-CAL-BLK = CNT-CAL-BLK + 1 .

Step 24: Continue counting the time until a new call arrives.

Step 25: Repeat steps 3 to 24 except steps 17 to 22 for no conversion of wavelength method and steps 3 to 24 except steps 15 and 16 for conversion of wavelength method.

Step 26: Blocking probability = CNT-CAL-BLK /Total number of calls.

Step 27: Throughput $=1.0-$ Blocking probability

2.2 Probability of Unnecessary Handover: The unnecessary handover probability can be derived for the case of call getting handed over unnecessarily to another network. The overall probability of call getting blocked is the product of unnecessary handover probability and blocking probability.

\section{$P_{\text {unsuccessful }}$ \\ = Unnecessary Handover Probabability $X$ Blocking Probability

UnnecessaryHandover Probability

$=P_{n 1} P_{n 2 / n 1} \sum_{j=L}^{B_{2}} \Pi_{2, B_{2}-j} \sum_{k=0}^{j-L} \Pi_{1, B_{1}-k} \xi_{1}(k, r, D)$

- $\sum_{i=0}^{B_{1}} \Pi_{1, B_{1}-i} \sum_{k=i+L}^{B_{2}} \Pi_{2, B_{2}-k} \psi_{2}(k, r, D)$

$+P_{n 1} P_{n 3 / n 1} \sum_{m=L}^{B_{3}} \Pi_{3, B_{3}-m} \sum_{k=0}^{m-L} \Pi_{1, B_{1}-k} \xi_{1}(k, r, D)$

- $\sum_{i=0}^{B_{1}} \prod_{1, B_{1}-i} \sum_{k=i+L}^{B_{3}} \Pi_{3, B_{3}-k} \psi_{3}(k, r, D)$

$+P_{n 2} P_{n 1 / n 2} \sum_{j=L}^{B_{1}} \prod_{1, B_{1}-j} \sum_{k=j-L+1+1}^{B_{1}} \prod_{1, B_{1}-k} \psi_{1}(k, r, D)$

- $\sum_{i=0}^{B_{2}} \Pi_{2, B_{2}-i} \sum_{k=0}^{i+L-1-1} \Pi_{2, B_{2}-k} \xi_{2}(k, r, D)$

$+P_{n 2} P_{n 3 / n 2} \sum_{j=L}^{B_{3}} \prod_{3, B_{3}-j} \sum_{k=j-L+1+1}^{B_{3}} \prod_{3, B_{3}-k} \psi_{3}(k, r, D)$

- $\sum_{m=0}^{B_{2}} \Pi_{2, B_{2}-m} \sum_{k=0}^{m+L-1-1} \Pi_{2, B_{2}-k} \xi_{2}(k, r, D)$

$+P_{n 3} P_{n 1 / n 3} \sum_{m=L}^{B_{1}} \prod_{1, B_{1}-m} \sum_{k=m-L+1}^{B_{1}} \Pi_{1, B_{1}-k} \psi_{1}(k, r, D)$

- $\sum_{i=0}^{B_{3}} \Pi_{3, B_{3}-i} \sum_{k=0}^{i+L-1-1} \Pi_{3, B_{3}-k} \xi_{3}(k, r, D)$

$+P_{n 3} P_{n 2 / n 3} \sum_{j=L}^{B_{2}} \prod_{2, B_{2}-j} \sum_{k=0}^{j-L} \prod_{3, B_{3}-k} \xi_{3}(k, r, D)$

- $\sum_{m=0}^{B_{3}} \prod_{3, B_{3}-m} \sum_{k=m+L}^{B_{2}} \prod_{2, B_{2}-k} \psi_{2}(k, r, D)$

The notations and definitions of the quantities expressed in Eq. 5 can be found in author's paper C P Mallikarjuna Gowda et. al (2018). 
III. Simulation Results: Blocking probabilities are derived for tandem networks with conversion and without conversion. The converters are used when there is a call blockage. The converters are assumed to be installed at all nodes. Both type of calls, namely, primary unit calls and secondary unit calls are treated in these simulations. When calls arrive, three types of assignment methods, such a first fit assignment, uniformly distributed random assignment and Selective channel assignment are used in these simulations to allocate a channel to the calls. Hence the following models based on type of channel assignment and conversion is developed.

PU-SU-NC-FF: First fit assignment method with no conversion and both types of calls

PU-SU-NC-RANDOM-ASSIGN: Random assignment method with no conversion and both types of calls

PU-NC-SEL-ASSIGN: Selective channel assignment method with no conversion and only PU calls

SU-NC-SEL-ASSIGN: Selective channel assignment method with no conversion and only SU calls

PU-SU-C-FF: First fit assignment method with conversion and both types of calls

PU-SU-C-RANDOM-ASSIGN: Random assignment method with conversion and both types of calls

PU-C-SEL-ASSIGN: Selective channel assignment method with conversion and only PU calls

SU-C-SEL-ASSIGN: Selective channel assignment method with conversion and only SU calls.

Figure 1: Blocking Probability of assignment methods for a load 15 Erlangs per link, 20 nodes, 15 Channels, 4000 iterations, and $70 \%$ of PU calls with no conversion.

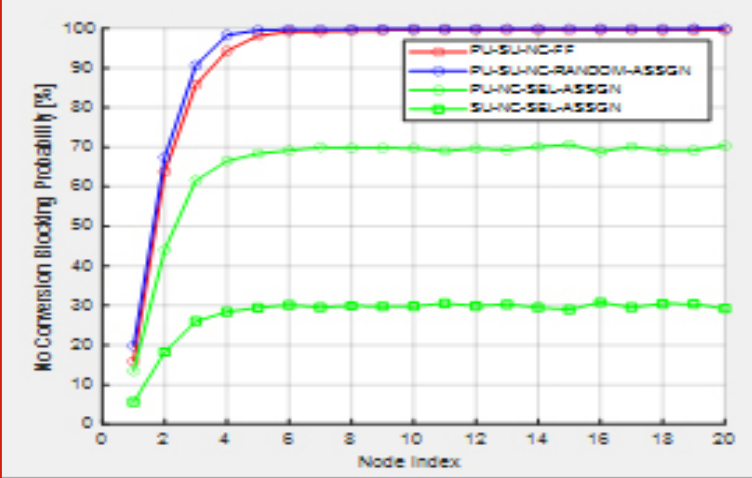

Fig. 1 shows the blocking probability of first fit assignment, uniformly distributed random assignment and selective channel assignment methods for a load 15 Erlangs per link and with 20 Links (nodes), 15 Channels, 4000 iterations and 70\% of PU calls with no conversion. It can be observed that blocking probabilities of selective channel assignment method is much lower than that of first fit assignment and uniformly distributed random assignment methods. The blocking probabilities of PU-NC-SEL-ASSIGN and SU-NC-SEL-ASSIGN are 70\% of PU-SU-NC-FF and PU-SU-NC-RANDOM-ASSIGN models. This is attributed to the reason that in selective channel assignment, the assignment is made near the channels towards the end of lower order (near channel 1) for PU calls and towards end of higher order (near channel 15) for SU calls.

Fig. 2 shows the channel utilization of first fit assignment, uniformly distributed random assignment and selective channel assignment methods for a load 15 Erlangs per link and with 20 Links (nodes), 15 Channels, 4000 iterations and 70\% of PU calls with no conversion. It can be noticed that in first fit (FF) assignment method, the only channels near lower order is assigned most. In case of random assignment (RANDOM-ASSGN) all channels are assigned uniformly. In case of selective channel (SEL-ASSGN) assignment, channels near 1 and 15 are assigned most.

Figure 2: Channel utilization of assignment methods for a load 15 Erlangs per link, 20 nodes, 15 Channels, 4000 iterations and 70\% of PU calls with no conversion.

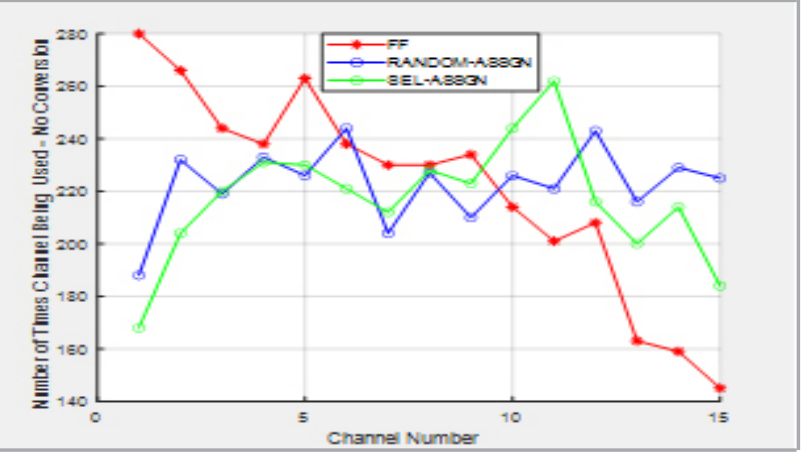

Figure 3: Blocking Probability of assignment methods for a load 15 Erlangs per link, 20 nodes, 15 Channels, 4000 iterations and $70 \%$ of PU calls with conversion.

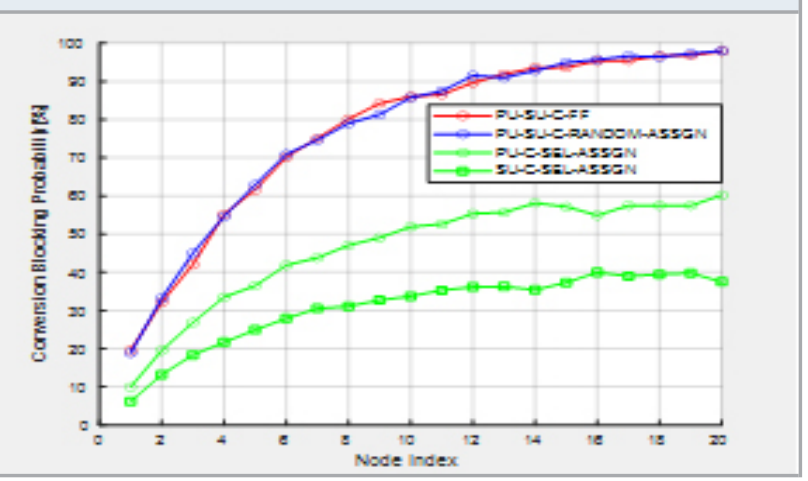

Fig. 3 shows the blocking probability of first fit assignment, uniformly distributed random assignment and selective channel assignment methods for a load 15 Erlangs per link, 20 nodes, 15 Channels, 4000 iterations and 70\% of PU calls with conversion. It can be observed in this case also, blocking probabilities of selective channel assignment method is much lower than that of first fit assignment and uniformly distributed random 
assignment methods. Similar to the no conversion models, in selective channel assignment, the assignment is made near the channels towards the end of lower order (near channel 1) for PU calls and towards end of higher order (near channel 15) for SU calls, whereas all the calls are assigned only near lower order in First fit and uniformly across all channels in random assignment methods. Hence the blocking probabilities of PU-CSEL-ASSIGN and SU-C-SEL-ASSIGN are nearly $60 \%$ of PU-SU-C-FF and PU-SU-C-RANDOM-ASSIGN models. It can also be observed that when conversion is used it does not matter if the first fit or random assignment methods are used since both yield almost similar blocking probabilities. This is due to the reason that call blockage is eliminated with the help of converters irrespective of assignment method.

Figure 4: Channel utilization of assignment methods for a load 15 Erlangs per link, 20 nodes, 15Channels, 4000 iterations and $70 \%$ of PU calls with conversion.

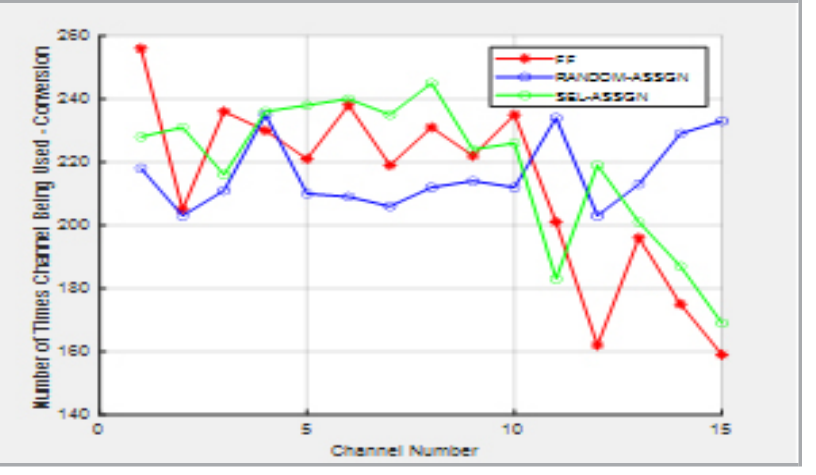

Figure 5: Blocking Probability of assignment methods for a load 15 Erlangs per link, 20 nodes, 15 Channels, 4000 iterations and $70 \%$ of PU calls with and without conversion.

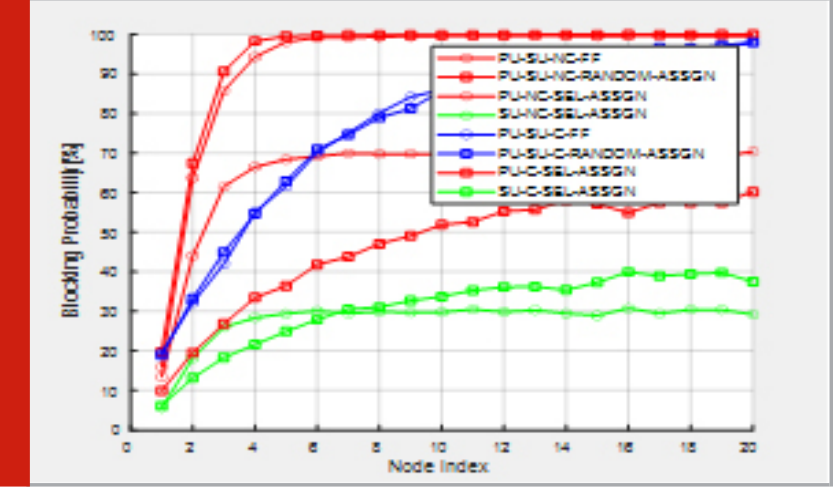

Fig. 4 shows the channel utilization of First fit assignment, uniformly distributed random assignment and Selective channel assignment methods for a load 15 Erlangs per link and with 20 Links (nodes), 15 Channels, 4000 iterations and $70 \%$ of PU calls with conversion. In this case also, the pattern of channel utilization is also most similar to that of no-conversion models. First fit (FF) assignment method has only channels near lower order assigned most and random assignment (RANDOMASSGN) has all channels assigned uniformly. In selective channel (SEL-ASSGN) assignment, channels towards the end of lower order and higher order are assigned most.

Figure 6: Throughput of assignment methods for a load 15 Erlangs per link, 20 nodes, 15 Channels, 4000 iterations and $70 \%$ of PU calls with and without conversion.

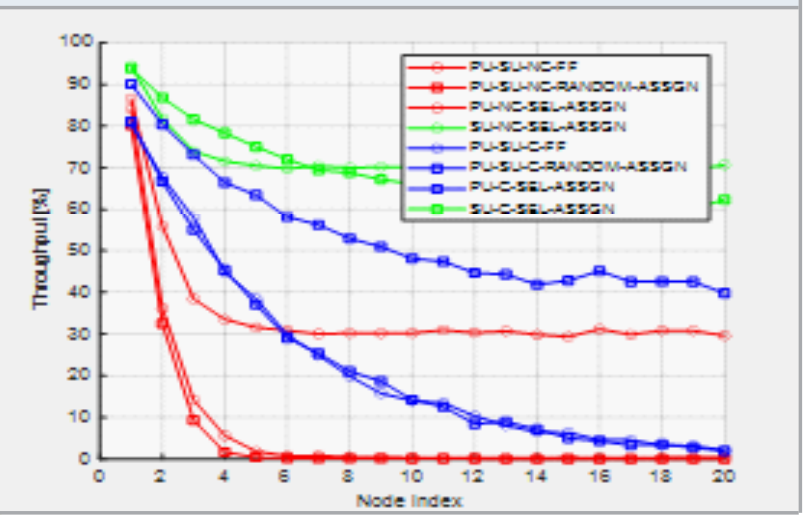

Fig. 5 and 6 shows the blocking probability and throughput, respectively, of First fit assignment, uniformly distributed random assignment and Selective channel assignment methods for a load 15 Erlangs per link and with 20 Links (nodes), 15 Channels, 4000 iterations and $70 \%$ of PU calls with and without conversion. Blocking probabilities of first fit assignment (PU-SU-NC-FF) is $99 \%$ at the node index 20 , whereas it is $100 \%$ at node 20 in case of uniformly distributed random assignment (PU-SU-NC-RANDOM-ASSIGN) method. These blocking probabilities can be reduced by using converters at all the nodes. The Blocking probabilities of first fit assignment (PU-SU-C-FF) are reduced to 94\% and to 94\% at node 20 in case of uniformly distributed random assignment (PU-SU-C-RANDOM-ASSIGN) method.

Figure 7: Blocking Probability of assignment methods for a load 15 Erlangs per link, 20 nodes, 15 Channels, 4000 iterations and $60 \%$ of PU calls with no conversion.

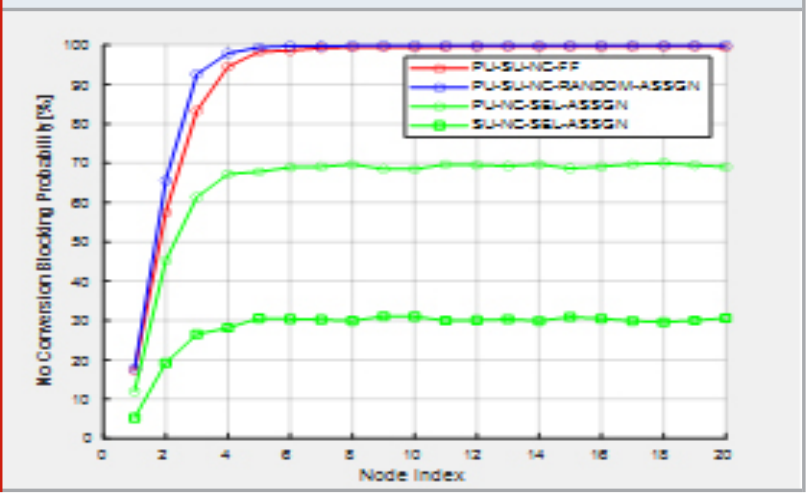

The blocking probabilities can be further reduced with and without converters using selective channels assignment. With no converters, the selective channel method (PU-NC-SEL-ASSIGN and SU-NC-SEL-ASSIGN) yields approximately $70 \%$ blocking probability and with conversion (PU-C-SEL-ASSIGN and SU-C-SEL-ASSIGN), it is approximately 50\% on average. 
Figure 8: Channel utilization of assignment methods for a load 15 Erlangs per link, 20 nodes, 15 Channels, 4000 iterations and $60 \%$ of PU calls with no conversion.

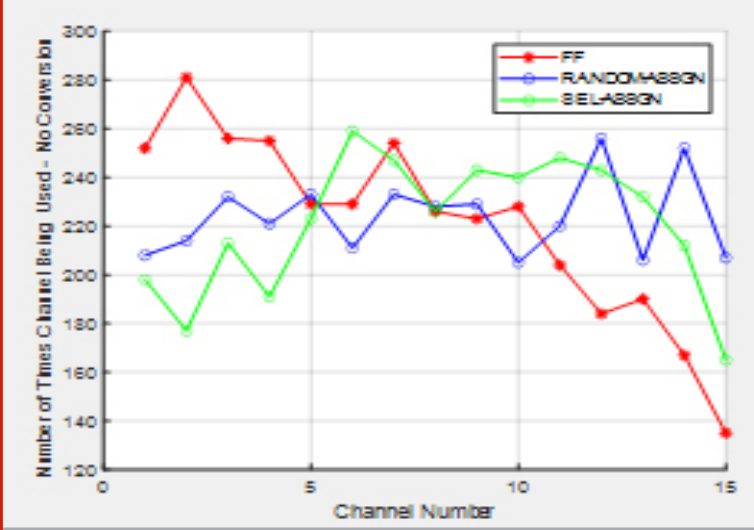

Figure 9: Blocking Probability of assignment methods for a load 15 Erlangs per link, 20 nodes, 15 Channels, 4000 iterations and $60 \%$ of PU calls with conversion.

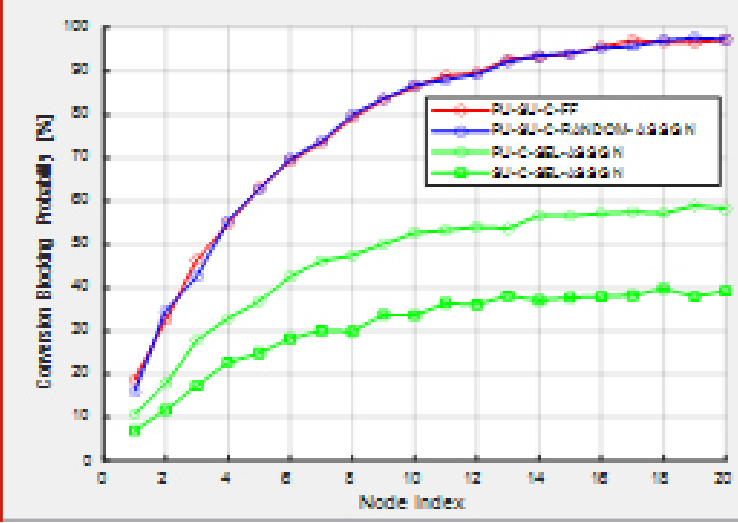

Fig.7 shows the blocking probability of First fit assignment, uniformly distributed random assignment and Selective channel assignment methods for a load 15 Erlangs per link and with 20 Links (nodes), 15 Channels, 4000 iterations and 60\% of PU calls with no conversion. When the PU calls are set at 70\% of total calls, the blocking probability for the PU calls is 70\% with PU-NC-SEL-ASSIGN and that for SU calls is 30\% with SU-NC-SEL-ASSIGN model. In case of PU-SU-NC-FF and PU-SU-NC-RANDOM-ASSIGN models, the blocking probabilities are near 99\% and 100\% respectively when no converters are used.

Fig.9 shows the blocking probability of First fit assignment, uniformly distributed random assignment and Selective channel assignment methods for a load 15 Erlangs per link and with 20 Links (nodes), 15 Channels, 4000 iterations and $60 \%$ of PU calls with conversion. When there are 60\% PU calls in the total calls, the blocking probabilities for the PU is usually higher than that of the SU calls. The same can be noticed in Fig. 7. With conversion, there is no difference between the first fit or random assignment methods. The blocking probabilities of PU-SU-C-FF and PU-SU-C-RANDOM-
ASSIGN are nearly $97 \%$ at the node 20 . When the selective assignment models are used, it is brought down to $60 \%$ and $40 \%$ respectively with PU-C-SEL-ASSIGN and SU-C-SEL-ASSIGN models.

Figure 10: Channel utilization of assignment methods for a load 15 Erlangs per link, 20 nodes, 15 Channels, 4000 iterations and $60 \%$ of PU calls with conversion.

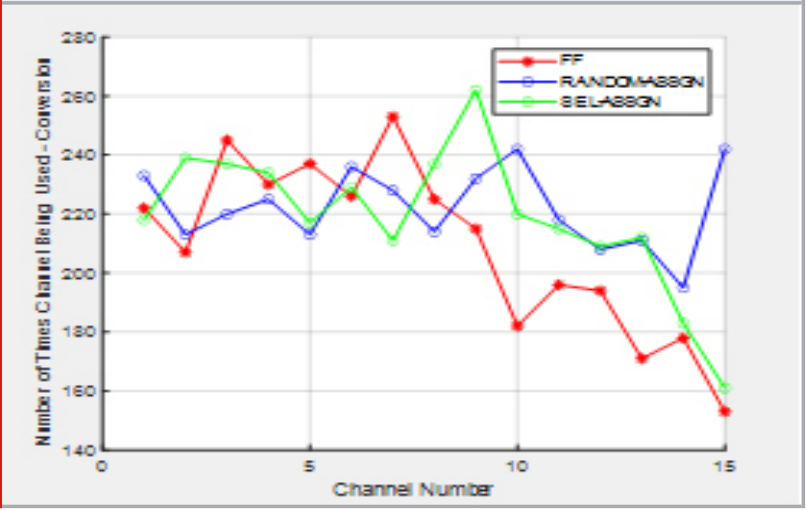

Figure 11: Blocking Probability of assignment methods for a load 15 Erlangs per link, 20 nodes, 15 Channels, 4000 iterations and $60 \%$ of PU calls with and without conversion.

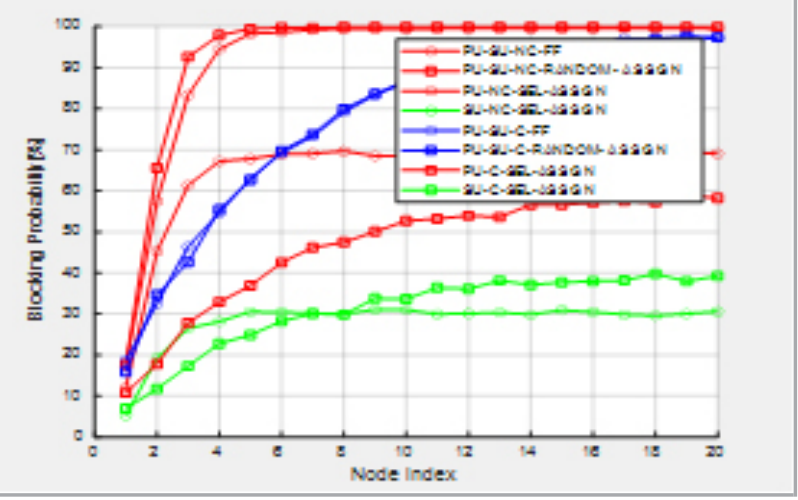

Figure 12: Throughput of assignment methods for a load 15 Erlangs per link, 20 nodes, 15 Channels, 4000 iterations and $60 \%$ of PU calls with and without conversion.

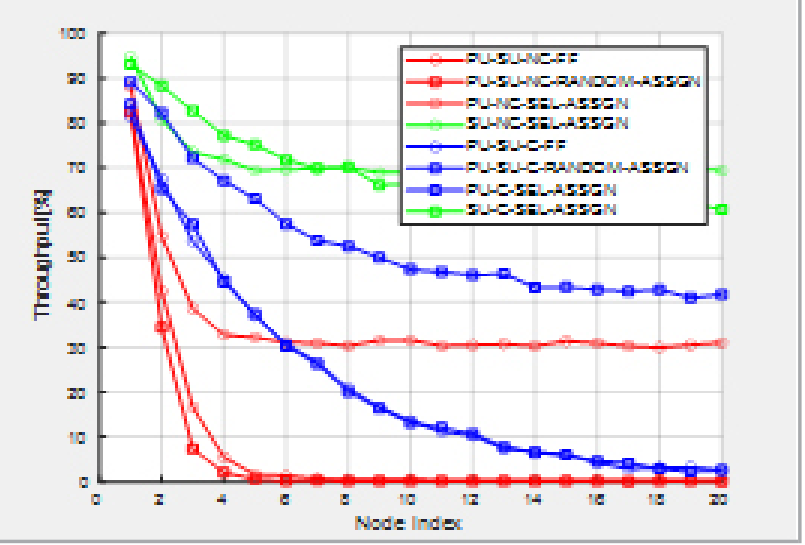


Table 1 . Summary of all the 8 models

\begin{tabular}{|c|c|c|}
\hline $\begin{array}{l}\text { Percentage } \\
\text { PU calls }\end{array}$ & Model & $\begin{array}{l}\text { Blocking prob. } \\
\text { at Node } 20\end{array}$ \\
\hline $60 \%$ & PU-SU-NC-FF & $100 \%$ \\
\hline $60 \%$ & $\begin{array}{c}\text { PU-SU-NC- } \\
\text { RANDOM-ASSIGN }\end{array}$ & $100 \%$ \\
\hline $60 \%$ & PU-NC-SEL-ASSIGN & $70 \%$ \\
\hline $60 \%$ & SU-NC-SEL-ASSIGN & $30 \%$ \\
\hline $60 \%$ & PU-SU-C-FF & $97 \%$ \\
\hline $60 \%$ & $\begin{array}{c}\text { PU-SU-C-RANDOM- } \\
\text { ASSIGN }\end{array}$ & $97 \%$ \\
\hline $60 \%$ & PU-C-SEL-ASSIGN & $60 \%$ \\
\hline $60 \%$ & SU-C-SEL-ASSIGN & $40 \%$ \\
\hline $70 \%$ & PU-SU-NC-FF & $100 \%$ \\
\hline $70 \%$ & $\begin{array}{c}\text { PU-SU-NC-RANDOM- } \\
\text { ASSIGN }\end{array}$ & $100 \%$ \\
\hline $70 \%$ & PU-NC-SEL-ASSIGN & $70 \%$ \\
\hline $70 \%$ & SU-NC-SEL-ASSIGN & $30 \%$ \\
\hline $70 \%$ & PU-SU-C-FF & $96 \%$ \\
\hline $70 \%$ & $\begin{array}{c}\text { PU-SU-C-RANDOM- } \\
\text { ASSIGN }\end{array}$ & $96 \%$ \\
\hline $70 \%$ & PU-C-SEL-ASSIGN & $60 \%$ \\
\hline $70 \%$ & SU-C-SEL-ASSIGN & $28 \%$ \\
\hline
\end{tabular}

Fig. 6 and 12 shows the blocking probability and throughput, respectively, of First fit assignment, uniformly distributed random assignment and Selective channel assignment methods for a load 15 Erlangs per link and with 20 Links (nodes), 15 Channels, 4000 iterations and 70\% of PU calls with and without conversion. The Fig. 12 shows the overall summary of all blocking probabilities with all the 8 models developed as part of this work. Blocking probabilities of first fit assignment (PU-SU-NC-FF) and of uniformly distributed random assignment (PU-SU-NC-RANDOM-ASSIGN) method are 99\% and 100\% respectively at the node index 20. Blocking probabilities could be brought down to $97 \%$ and to $95 \%$ at node 20 by using converters at all the nodes with first fit assignment (PU-SU-C-FF) and uniformly distributed random assignment (PU-SU-CRANDOM-ASSIGN) methods.

When selective channel assignment methods are used, the blocking probability is 70\% with PU-NC-SEL-ASSIGN model and 30\% with SU-NC-SEL-ASSIGN model. With conversion, that is when PU-C-SEL-ASSIGN and SU-CSEL-ASSIGN are used it yielded approximately $60 \%$ and $40 \%$ of blocking probability respectively. Throughput for all the above discussed models are shown in the fig 12 and it is found that the throughput of the proposed method i.e., selective channel assignment method is superior than the considered existing methods.

From the summary presented in Table 1 , the selective channel assignment methods, namely, PU-C-SEL-
Figure 13: UHP Vs occupied channels for B1 $=15, \mathrm{~B} 2=15$ and $\mathrm{B} 3=15$.

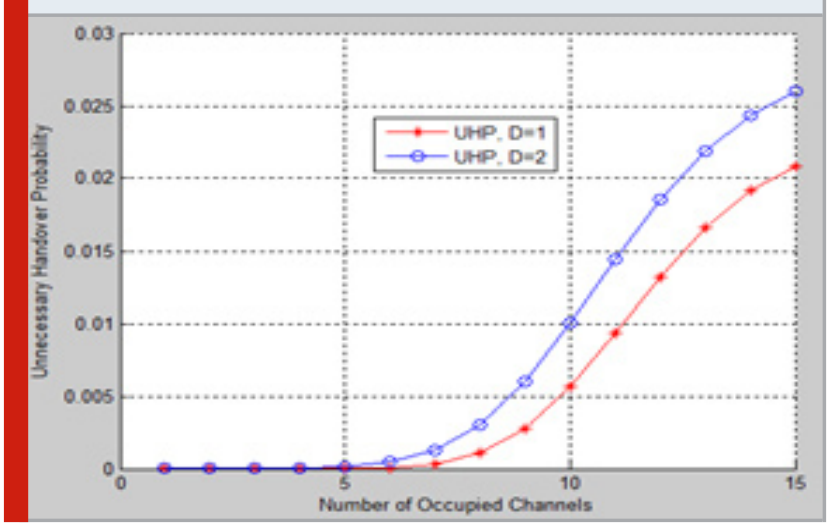

ASSIGN and SU-C-SEL-ASSIGN yield the best results both for $60 \%$ and $70 \%$ PU call cases.

\section{CONCLUSION}

In this paper, authors have developed the models to simulate for the blocking probabilities with conversion and without conversion. Also, the percentage of PU calls over all the calls is varied. Two cases, one with 60\% PU calls and 70\% PU calls are simulated. The assignment methods, namely, first fit assignment, uniformly distributed random assignment and Selective channel assignment methods are used in the simulations. It has been observed that the blocking probabilities are nearly $100 \%$ when the first fit and random assignment methods are used for a network with 15 channels, 20 links and 15 Erlangs. of load.

This is true for both 60\% and 70\% PU calls. When the selective channel assignment method was used, the blocking probabilities are around 70\% and 30\% for 60\% PU calls case and 70\% and 30\% for 70\% PU calls case when there were no converters in the network. When converters are used, the blocking probabilities are around $60 \%$ and $40 \%$ for $60 \%$ PU calls case and $60 \%$ and $28 \%$ for $70 \%$ PU calls case. Hence it is concluded that selective assignment method has outperformed over the first fit assignment and uniformly distributed channel assignment methods in conversion and no conversion as well as $60 \%$ and $70 \%$ of PU calls.

\section{REFERENCES}

Amir Sepasi Zahmati, Xavier Fernando and Ali Grami (2009), "Steady-State Markov Chain Analysis for Heterogeneous Cognitive Radio Networks”, IEEE, ICC, proceedings.

C P Mallikarjuna Gowda and T Vijayakumar (2018) "A New Channel Assignment Method in Cognitive Radio System", ICTACT Journal on Communication Technology, 09(04): 1885-1892.

C P Mallikarjuna Gowda and T Vijayakumar (2018), "Blocking Probabilities, Resource Allocation Problems and Optimal Solutions in Cognitive Radio Networks: 
A Survey", 3rd International Conference on Electrical, Electronics, Communication, Computer Technologies and Optimization Techniques.: 1493-1498.

G. Cao and M. Singhal (2000), "Distributed fault-tolerant channel allocation for cellular networks", IEEE Journal of Selected Areas in Communications, 18(7):13261337.

Haythem Bany Salameh, Reema Qawasmeh, and Ahmad F. Al-Ajlouni (2020), "Routing with Intelligent Spectrum Assignment in Full-Duplex Cognitive Networks Under Varying Channel Conditions", IEEE Communications Letters, 24(4): 872-876.

H. Jiang and S. Rappaport (1996), "Prioritized channel borrowing without locking: a channel sharing strategy for cellular communications", IEEE/ACM Transactions on Networking, 4(2): 163-172.

I. Ketzela, and M. Nagheshineh (1996), "Channel Assignment Schemes for Cellular Mobile Telecommunication Systems: A Comprehensive Survey", IEEE Personal Communications, 3(3): 10-31.

J.C.-I. Chuang (1993), "Performance issues and algorithms for dynamic channel assignment”, IEEE J. Selected Areas in Comm., 11(6): 955-963.

Muhammad Rehan Usman, Muhammad Arslan Usman and Soo Young Shin (2017), “Channel Blocking Analysis and Availability Prediction in Cognitive Radio Networks", Workshop on Computing, Networking and Communications (CNC).

Murtaza Zafer and Eytan Modiano (2006), "Blocking Probability and Channel Assignment in Wireless Networks", IEEE Transactions on Wireless Communications, 5(4): 1-11.

Rajeev Ranjan, Navneet Agrawal, Sunil Joshi (2020), "Interference mitigation and capacity enhancement of cognitive radio networks using modified greedy algorithm/channel assignment and power allocation techniques”, IET Communications, 14(9): 1502-1509.

R. Kaniezhil and Dr. C. Chandrasekar (2012), "Multiple Service providers sharing Spectrum using Cognitive Radio", International Journal of Scientific \&t Engineering Research, 3(3): 1-7.

R. Kaniezhil, Dr. C. Chandrasekar, S. Nithya Rekha
(2012), "Channel Selection for Selective Sensing using Cognitive Radio Nodes", International Journal of Computer Applications, 39(3): 20-25.

R. Mathar and J. Mattfeldt (1993), "Channel assignment in cellular radio networks”, IEEE Trans. on Veh. Technol, 42(4): 647-656.

Sandeep Mavuduru Kannappa and Mohammad Saquib (2010), "Performance Analysis of a Cognitive Network with Dynamic Spectrum Assignment to Secondary Users", IEEE, ICC proceedings.

S.K. Das, S.K. Sen and R. Jayaram (1996), "A dynamic load balancing strategy for channel assignment using selective borrowing in cellular mobile environment, in", in Proc. of IEEE/ACM Conf. on Mobile Computing and Networking . : 73-84.

S. A. El-Dolil, W. C. Wong, and R. Steele (1989), "Teletraffic performance of highway microcells with overlay macrocell”, IEEE J. Sel. Areas Communication. 7(1): 71-78.

Vamsi Krishna Tumuluru, Ping Wang, Dusit Niyato, and Wei Song (2013), "Performance Analysis of Cognitive Radio Spectrum Access with Prioritized Traffic”, IEEE Transactions on Vehicular Technology: 1-11.

Xiukui Li and Seyed A. (Reza) Zekavat (2009), "Traffic Pattern Prediction Based Spectrum Sharing for Cognitive Radios”, Lecture notes in Electrical and Electronic Engineering, ISBN 978-953-307-021-6.

X. Li S.A. (Reza) Zekavat (2010), "Spectrum sharing across multiple service providers via cognitive radio nodes", IET Communication, 4(5) : 551-561.

Yan Zhang (2008), "Dynamic Spectrum Access in Cognitive Radio Wireless Networks”, IEEE, ICC proceedings: 4927-4932.

Yousuf Aborahama and Mohamed S. Hassan (2020), "On the Stochastic Modeling of the Holding Time of SUs to PU Channels in Cognitive Radio Networks", IEEE Transactions on Cognitive Communications and Networking, 6(1): 282-295.

Zeljko Tabakovic and Mislav Grgic (2016), “Cognitive radio frequency assignment with interference weighting and categorization", EURASIP Journal on Wireless Communications and Networking, 2016:45. 